PROCEEDINGS OF THE

AMERICAN MATHEMATICAL SOCIETY

Volume 135, Number 10, October 2007, Pages 3393-3402

S 0002-9939(07)09050-8

Article electronically published on June 22, 2007

\title{
SOME 3-MANIFOLDS AND 3-ORBIFOLDS WITH LARGE FUNDAMENTAL GROUP
}

\author{
MARC LACKENBY
}

(Communicated by Daniel Ruberman)

\begin{abstract}
We provide two new proofs of a theorem of Cooper, Long and Reid which asserts that, apart from an explicit finite list of exceptional manifolds, any compact orientable irreducible 3-manifold with non-empty boundary has large fundamental group. The first proof is direct and topological; the second is group-theoretic. These techniques are then applied to prove a string of results about (possibly closed) 3-orbifolds, which culminate in the following theorem. If $K$ is a knot in a compact orientable 3-manifold $M$ such that the complement of $K$ admits a complete finite-volume hyperbolic structure, then the orbifold obtained by assigning a singularity of order $n$ along $K$ has large fundamental group for infinitely many positive integers $n$. We also obtain information about this set of values of $n$. When $M$ is the 3 -sphere, this has implications for the cyclic branched covers over the knot. In this case, we may also weaken the hypothesis that the complement of $K$ is hyperbolic to the assumption that $K$ is non-trivial.
\end{abstract}

\section{INTRODUCTION}

One of the key unresolved problems in 3-manifold theory is the Virtually Haken Conjecture. This asserts that if $M$ is a closed orientable hyperbolic 3-manifold, then $M$ has a finite-sheeted cover containing a properly embedded orientable incompressible surface (other than a 2-sphere). There is a range of stronger forms of this conjecture, possibly the strongest of which proposes that the fundamental group of $M$ is 'large'. This means that it has a finite index subgroup that admits a surjective homomorphism onto a non-abelian free group. The covering space of $M$ corresponding to such a finite index subgroup contains the required incompressible surface. Large groups have many other nice properties. For example, they have finite index subgroups with arbitrarily large first Betti number. In this paper, we will exhibit several classes of 3-manifolds and 3-orbifolds with large fundamental group. We start with a rapid and surprisingly elementary proof of the following theorem of Cooper, Long and Reid.

Theorem 2.1 ([5]). Let $M$ be a compact orientable irreducible 3-manifold with nonempty boundary. Then, either $M$ is an I-bundle over a surface with non-negative Euler characteristic or $\pi_{1}(M)$ is large.

Received by the editors May 12, 2006.

2000 Mathematics Subject Classification. Primary 57N10, 57M25.

The author was supported by the EPSRC.

(C)2007 American Mathematical Society Reverts to public domain 28 years from publication 
The original proof by Cooper, Long and Reid relied on some delicate and complicated 3-dimensional techniques. However, their aim was somewhat different from ours. They first showed that a compact orientable hyperbolic 3-manifold $M$ with non-empty boundary (other than an $I$-bundle over a surface) has a finite-sheeted cover that contains a closed embedded orientable incompressible surface (other than a 2-sphere) that is not boundary parallel. Further work was then required to ensure that this surface is non-separating, and yet more work was necessary to find two such surfaces whose union is non-separating. This then implies that $\pi_{1}(M)$ is large. Establishing these results was hard work, particularly when $\partial M$ does not consist entirely of tori. However, if one is content solely with proving that $\pi_{1}(M)$ is large, without establishing the existence of a closed essential surface in some finite cover, then most of the difficult 3-dimensional arguments can be sidestepped, as we shall see.

It is interesting to note that, in fact, one can almost entirely dispense with the topology and prove Theorem 2.1 using primarily group-theoretic techniques (at least in the main case, when $M$ is hyperbolic). We supply such a proof, which uses a recent theorem of the author, that gives a criterion for a finitely presented group to be large, in terms of the behaviour of its finite index subgroups 9. However, there is still topology lurking in the background, as this largeness criterion was proved using topological methods.

One of the main reasons why the topological proof of Theorem 2.1 is useful is that it naturally extends to certain 3-orbifolds. We pursue this line of investigation in Section 3 and give a brief description of these results below. These provide new classes of 3-orbifolds (and hence 3-manifolds) that have large fundamental groups.

In this paper, we assume some familiarity with the basic theory of 3-orbifolds. In particular, we take as given the following terminology: singular locus, covering space, fundamental group. We denote both the usual fundamental group of a space and the (orbifold) fundamental group of an orbifold by $\pi_{1}($ ). For an explanation of these terms and an introduction to orbifolds, we suggest [4] as a helpful reference.

Throughout this paper, an orbifold is allowed to have an empty singular locus and hence to be a manifold. If $O$ is a 3 -orbifold and $L$ is a link in $O$ disjoint from the singular locus and $n$ is a positive integer, then we denote by $O(L, n)$ the orbifold obtained from $O$ by adding singularities along $L$ of order $n$. We will prove a sequence of results about 3 -orbifolds, which lead to the following.

Theorem 3.6. Let $O$ be a compact orientable 3-orbifold (with possibly empty singular locus), and let $K$ be a knot in $O$, disjoint from its singular locus, such that $O-K$ has a complete, finite volume hyperbolic structure. Then, for infinitely many values of $n, \pi_{1}(O(K, n))$ is large.

Setting $O$ to be the 3 -sphere in Theorem 3.6, this applies to the much studied case of cyclic branched covers over hyperbolic classical knots. In fact, by use of the Orbifold Theorem and applying results from Section 3, we can obtain the following information about branched covers over any non-trivial knot in the 3-sphere.

Theorem 3.7. Let $K$ be a non-trivial knot in the 3-sphere, and let $m$ be any integer more than two. Then, for all sufficiently large $n$, the $m n$-fold cyclic cover of $S^{3}$ branched over $K$ has large fundamental group. 
Some parts of this paper present new proofs of known results; other bits give new theorems. However, the outstanding paper of Cooper, Long and Reid [5] has exerted a strong influence throughout.

Another simple proof of Theorem 2.1 has appeared recently, due to Button [3]. He showed that it can be deduced quite quickly from Howie's criterion [7 for a group to be large. Ratcliffe also established largeness in the case where $M$ has a boundary component with genus at least two in 14, providing a very quick proof based on a theorem of Baumslag and Pride [1].

\section{BOUNDED 3-MANIFOLDS}

Our goal in this section is to provide two new proofs of the following theorem.

Theorem 2.1 ([5]). Let $M$ be a compact orientable irreducible 3-manifold with nonempty boundary. Then, either $M$ is an I-bundle over a surface with non-negative Euler characteristic or $\pi_{1}(M)$ is large.

The principle reason why compact orientable 3-manifolds with non-empty boundary are more tractable than closed manifolds is the following result. This gives a lower bound on the rank of their cohomology in terms of the genus of their boundary. This result is a well known consequence of Poincaré duality.

Theorem 2.2. Let $M$ be a compact orientable 3-manifold, and let $i: P \rightarrow M$ be the inclusion of a compact (possibly empty) subsurface of $\partial M$. Then

$$
\operatorname{rank}\left(\operatorname{ker}\left(i^{*}: H^{1}(M) \rightarrow H^{1}(P)\right)\right) \geq \frac{1}{2} b_{1}(\partial M)-b_{1}(P) .
$$

To establish largeness, we will use the following well known lemma.

Lemma 2.3. Let $M$ be a compact 3-manifold. Suppose that $M$ contains two disjoint, transversely oriented, properly embedded surfaces whose union is nonseparating. Then $\pi_{1}(M)$ admits a surjective homomorphism onto $\mathbb{Z} * \mathbb{Z}$.

Proof. Let $S_{1}$ and $S_{2}$ be the two surfaces. There is the following collapsing map $f: M \rightarrow S^{1} \vee S^{1}$. The restriction of $f$ to a regular neighbourhood $N\left(S_{i}\right)$ is the composition of the homeomorphism $N\left(S_{i}\right) \rightarrow S_{i} \times I$ with projection onto the $I$ factor, followed by the quotient map from $I$ to the circle that glues the ends of the interval together, composed with the inclusion into the $i$ th circle of $S^{1} \vee S^{1}$. The map sends the remainder of $M$ to the central vertex of $S^{1} \vee S^{1}$. Fix a basepoint in $M$ disjoint from $N\left(S_{1}\right) \cup N\left(S_{2}\right)$. It is clear that $f_{*}: \pi_{1}(M) \rightarrow \mathbb{Z} * \mathbb{Z}$ is a surjection, because any element of $\mathbb{Z} * \mathbb{Z}$ may be realised by a based loop in $M$.

Proof of Theorem 2.1. First suppose that $\partial M$ contains a 2-sphere. Then, by irreducibility, $M$ is a 3 -ball, which is an $I$-bundle over a disc, verifying the theorem in this case. Thus, we may assume that each component of $\partial M$ has genus at least one.

Consider first the case where each component of $\partial M$ is a torus. A standard argument then allows us to assume that $M$ is hyperbolic. This argument can be found in Cooper, Long and Reid's paper [5, but we repeat it here for the sake of completeness. When $M$ is not hyperbolic, Thurston's geometrisation theorem [13] implies that $M$ is either Seifert fibred or contains an essential embedded torus. In 
the former case, the argument divides according to whether the base orbifold of the Seifert fibration has positive, zero or negative Euler characteristic. When it is positive, the manifold is a solid torus, which is an $I$-bundle over an annulus. When it is zero, the manifold again admits some $I$-bundle structure over a torus or Klein bottle. When the base orbifold has negative Euler characteristic, it has a finite-sheeted cover which is an orientable surface also with negative Euler characteristic. This induces a finite covering $\tilde{M} \rightarrow M$. The Seifert fibration on $\tilde{M}$ induces a surjective homomorphism from $\pi_{1}(\tilde{M})$ onto the fundamental group of this surface, which then admits a surjective homomorphism onto a free non-abelian group. Hence, when $M$ is Seifert fibred, it satisfies the conclusion of the theorem.

If $M$ contains an essential embedded torus, then it is a theorem of Kojima 8] and Luecke 12 (see also the work of Niblo and Long in [10] and 11]) that either $\pi_{1}(M)$ is large or $M$ is finitely covered by a torus bundle over the circle, the torus lifting to a fibre. The latter case cannot arise since $M$ has non-empty boundary.

We may therefore assume (when $\partial M$ consists entirely of tori) that the interior of $M$ is a complete, finite-volume hyperbolic 3-manifold. A lemma of Cooper, Long and Reid (Lemma 2.1 of [5]) asserts that, by passing to a finite-sheeted covering space if necessary, we may assume that $M$ has at least three boundary components.

All the above follows the argument of Cooper, Long and Reid, but here our proofs diverge. We have reached the stage where either $\partial M$ consists of tori and there are at least three of these or $\partial M$ has a component with genus at least two. In the former case, set $P$ to be one of these tori; otherwise let $P$ be the empty set. Then Theorem 2.2 gives that the kernel of $i^{*}: H^{1}(M) \rightarrow H^{1}(P)$ has rank at least $\frac{1}{2} b_{1}(\partial M)-b_{1}(P)$, which is positive. Let $\alpha$ be a non-trivial primitive element in the kernel of $i^{*}: H^{1}(M) \rightarrow H^{1}(P)$. Let $S$ be a properly embedded oriented surface in $M$, disjoint from $P$, dual to $\alpha$. We may assume that $S$ intersects each toral component of $\partial M$ in a (possibly empty) collection of coherently oriented essential curves. Now, $\alpha$ induces a surjective homomorphism $\pi_{1}(M) \rightarrow \mathbb{Z}$. Composing this with the homomorphism $\mathbb{Z} \rightarrow \mathbb{Z} / n \mathbb{Z}$ that reduces modulo $n$, we obtain a homomorphism $\pi_{1}(M) \rightarrow \mathbb{Z} / n \mathbb{Z}$. Let $M_{n}$ be the corresponding $n$-fold cyclic cover of $M$. This contains $n$ disjoint copies of $S$ which can be labelled with the integers modulo $n$. Let $F_{n}$ be the union of the surface with label 0 and the surface with label $\lfloor n / 2\rfloor$. This is a separating surface, dividing $M_{n}$ into two 3-dimensional submanifolds, which we will call $A_{n}^{1}$ and $A_{n}^{2}$.

When $\partial M$ consists only of tori, $A_{n}^{1}$ and $A_{n}^{2}$ each contain at least $\lfloor n / 2\rfloor$ copies of $P$. Hence, for $j=1$ and $2, b_{1}\left(\partial A_{n}^{j}\right) \rightarrow \infty$ as $n \rightarrow \infty$. When $\partial M$ contains a non-toral component, the same is true, since $\partial A_{n}^{j}$ consists of $F_{n}$ and at least $\lfloor n / 2\rfloor$ copies of $\partial M$ cut along $S$, with their boundary components glued in pairs. However, in both cases, $b_{1}\left(F_{n}\right)$ remains independent of $n$. Therefore, when $n$ is sufficiently large, $\frac{1}{2} b_{1}\left(\partial A_{n}^{j}\right)>b_{1}\left(F_{n}\right)$ for both $j=1$ and 2. Let us fix an integer $n$ where these inequalities hold. Theorem 2.2 then gives that the kernel of $i^{*}: H^{1}\left(A_{n}^{j}\right) \rightarrow H^{1}\left(F_{n}\right)$ is non-trivial. Let $W_{n}^{j}$ be a connected oriented surface, properly embedded in $A_{n}^{j}$, disjoint from $F_{n}$, dual to a non-trivial primitive class in this kernel. (See Figure 1 for the case where $\partial M$ is a union of tori.) Then $W_{n}^{1}$ and $W_{n}^{2}$ are disjoint oriented surfaces the union of which is non-separating in $M_{n}$. By Lemma 2.3, $\pi_{1}(M)$ is large.

We now provide an alternative group-theoretic proof of Theorem 2.1 that uses the following recent theorem of the author (Theorem 1.2 of [9]). 


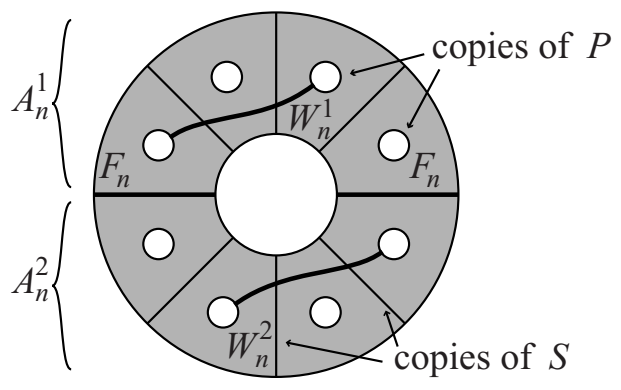

FIGURE 1.

Theorem 2.4. Let $G$ be a finitely presented group, and suppose that, for each natural number $n$, there is a triple $H_{n} \geq J_{n} \geq K_{n}$ of finite index normal subgroups of $G$ such that

(i) $H_{n} / J_{n}$ is abelian for all $n$;

(ii) $\lim _{n \rightarrow \infty}\left(\left(\log \left[H_{n}: J_{n}\right]\right) /\left[G: H_{n}\right]\right)=\infty$;

(iii) $\lim \sup _{n}\left(d\left(J_{n} / K_{n}\right) /\left[G: J_{n}\right]\right)>0$.

Then $K_{n}$ admits a surjective homomorphism onto a free non-abelian group, for infinitely many $n$.

Here, $d()$ denotes the rank of a group, which is the minimal size of a generating set.

Alternative proof of Theorem 2.1. As before, we may assume either that $\partial M$ consists entirely of tori and there are at least three of these or that $\partial M$ contains a higher genus component. Let $M_{n}$ be as in the previous proof.

We now wish to apply Theorem 2.4. Let $G$ be $\pi_{1}(M)$, and set $H_{n}$ to be $G$, for each $n$. Let $J_{n}$ be the subgroup of $G$ corresponding to the cover $M_{n}$, and set $K_{n}$ to be $J_{n}^{2}$, the subgroup generated by the squares of elements in $J_{n}$. We must check the various conditions of Theorem 2.4. Now, $J_{n}$ is a normal subgroup of $G$ by construction. As $K_{n}$ is a characteristic subgroup of $J_{n}$, it is therefore also normal in $G$. Now, $H_{n} / J_{n}$ is isomorphic to $\mathbb{Z} / n \mathbb{Z}$. In particular, it is abelian, verifying (i), and its order tends to infinity which gives (ii). Finally, $J_{n} / K_{n}$ is isomorphic to $H_{1}\left(M_{n} ; \mathbb{Z} / 2 \mathbb{Z}\right)$, which has rank at least $n$, by Theorem 2.2. Therefore, $d\left(J_{n} / K_{n}\right) /\left[G: J_{n}\right] \geq 1$, for each $n$, which gives (iii). Hence, by Theorem $2.4, \pi_{1}(M)$ is large.

\section{ORbifolds With LARGE FUNDAMENTAL GROUP}

In this section, we show that the techniques in the topological proof of Theorem 2.1 can be applied to 3-orbifolds. This is because we dealt in Section 2 with cyclic covers of large degree, and orbifolds also have have such covers, provided their singularities has sufficiently large singularity order.

We start with the following theorem, where the orbifold contains at least three distinguished components of its singular locus. All subsequent theorems will follow from this result. 
Theorem 3.1. Let $O$ be a compact orientable 3 -orbifold, and let $L$ be a link in $O$, disjoint from the singular locus of $O$, with at least three components. Then, for all sufficiently large $n, \pi_{1}(O(L, n))$ is large.

Proof. Let $|O|$ denote the underlying manifold of $O$. The natural map $O \rightarrow|O|$ induces a surjective homomorphism $\pi_{1}(O(L, n)) \rightarrow \pi_{1}(|O|(L, n))$. Hence, if the latter group is large, then so is the former. It therefore suffices to consider the case where $O$ is a manifold $M$, say.

Let $X$ denote the 3-manifold $M-\operatorname{int}(N(L))$. Pick a component $L_{1}$ of $L$. Since $L$ has at least three components, Theorem 2.2 implies that $i^{*}: H^{1}(X) \rightarrow H^{1}\left(\partial N\left(L_{1}\right)\right)$ has non-trivial kernel. Let $S$ be a properly embedded, connected, oriented surface in $X$ disjoint from $\partial N\left(L_{1}\right)$ dual to a non-trivial primitive element in this kernel. We may assume that $S$ intersects each toral component of $\partial X$ in a (possibly empty) collection of coherently oriented essential curves. Let $X_{n} \rightarrow X$ denote the associated $n$-fold cyclic cover of $X$. This extends to a cover $O_{n} \rightarrow M(L, n)$. Note that there is an inclusion map $X_{n} \rightarrow O_{n}$. There are $n$ copies of $S$ in $X_{n}$ which we may label with the integers modulo $n$. Let $F_{n}$ be the union of the surfaces labelled 0 and $\lfloor n / 2\rfloor$. Then $F_{n}$ divides $X_{n}$ into two manifolds $A_{n}^{1}$ and $A_{n}^{2}$. Each contains at least $\lfloor n / 2\rfloor$ copies of $\partial N\left(L_{1}\right)$. For $j=1$ and 2 , let $P_{n}^{j}$ be the copy of $F_{n}$ in $A_{n}^{j}$, together with any adjacent annuli of $\partial A_{n}^{j}-F_{n}$. Then $b_{1}\left(P_{n}^{j}\right)$ is independent of $n$. So, Theorem 2.2 implies that, when $n$ is sufficiently large, $i^{*}: H^{1}\left(A_{n}^{j}\right) \rightarrow H^{1}\left(P_{n}^{j}\right)$ has non-trivial kernel, for $j=1$ and 2 . Let $W_{n}^{j}$ be a connected properly embedded oriented surface in $A_{n}^{j}$, disjoint from $P_{n}^{j}$, dual to a primitive class in this kernel. Then $W_{n}^{1}$ and $W_{n}^{2}$ are disjoint oriented surfaces, the union of which is non-separating in $X_{n}$. Therefore, by Lemma 2.3, there is a surjective homomorphism $\pi_{1}\left(X_{n}\right) \rightarrow \mathbb{Z} * \mathbb{Z}$. Now, the inclusion map $X_{n} \rightarrow O_{n}$ induces a surjective homomorphism $\pi_{1}\left(X_{n}\right) \rightarrow \pi_{1}\left(O_{n}\right)$. There is an obvious surjective homomorphism $\mathbb{Z} * \mathbb{Z} \rightarrow(\mathbb{Z} / n \mathbb{Z}) *(\mathbb{Z} / n \mathbb{Z})$ that respects the free factors. We claim that $\pi_{1}\left(X_{n}\right) \rightarrow \mathbb{Z} * \mathbb{Z}$ descends to a surjective homomorphism $\pi_{1}\left(O_{n}\right) \rightarrow(\mathbb{Z} / n \mathbb{Z}) *(\mathbb{Z} / n \mathbb{Z})$. To prove this, we must show that the kernel of $\pi_{1}\left(X_{n}\right) \rightarrow \pi_{1}\left(O_{n}\right)$ is sent to the identity in $(\mathbb{Z} / n \mathbb{Z}) *(\mathbb{Z} / n \mathbb{Z})$. Now, this kernel is normally generated by powers of the meridian curves that encircle the singular locus of $O_{n}$, each power being the order of the relevant singular component. The meridian of any singular component not adjacent to $W_{n}^{1}$ or $W_{n}^{2}$ is sent to the identity under $\pi_{1}\left(X_{n}\right) \rightarrow \mathbb{Z} * \mathbb{Z}$. Each component of the singular locus adjacent to $W_{n}^{1}$ or $W_{n}^{2}$ has order $n$, and the $n$th power of its meridian is sent to the $n$th power of one of the free generators of $\mathbb{Z} * \mathbb{Z}$, which is in the kernel of $\mathbb{Z} * \mathbb{Z} \rightarrow(\mathbb{Z} / n \mathbb{Z}) *(\mathbb{Z} / n \mathbb{Z})$. Thus, the claim is verified: there is an induced surjective homomorphism $\pi_{1}\left(O_{n}\right) \rightarrow(\mathbb{Z} / n \mathbb{Z}) *(\mathbb{Z} / n \mathbb{Z})$. But $(\mathbb{Z} / n \mathbb{Z}) *(\mathbb{Z} / n \mathbb{Z})$ has a free non-abelian subgroup of finite index, provided $n>2$. Therefore, $\pi_{1}\left(O_{n}\right)$ is large, and so the same is true for $\pi_{1}(M(L, n))$, and hence $\pi_{1}(O(L, n))$.

The following result allows us to consider some 3-orbifolds with just one distinguished component of their singular locus.

Theorem 3.2. Let $O$ be a compact orientable 3-orbifold, and let $K$ be a knot in $O$ disjoint from the singular set of $O$. Suppose that there is a surjective homomorphism $\phi$ from $\pi_{1}(O)$ onto some finite group $H$, so that $\phi(\langle[K]\rangle)$ has index at least 3 in $H$. Then, provided $n$ is sufficiently large, $\pi_{1}(O(K, n))$ is large. 
Here, $[K]$ is some element of $\pi_{1}(O)$ representing $K$, and $\langle[K]\rangle$ is the subgroup generated by $[K]$. This is only defined up to conjugacy in $\pi_{1}(O)$, but the index of $\phi(\langle[K]\rangle)$ in $H$ is nevertheless well-defined.

Proof of Theorem 3.2. The kernel of $\phi$ corresponds to a finite covering $\tilde{O} \rightarrow O$. Let $L$ be the inverse image of $K$ in $\tilde{O}$. We then have an induced finite covering $\tilde{O}(L, n) \rightarrow O(K, n)$. The number of components of $L$ is equal to the index of $\phi(\langle[K]\rangle)$ in $H$, which we are assuming is at least 3. Hence, by Theorem 3.1, $\pi_{1}(\tilde{O}(L, n))$ is large for all sufficiently large $n$, and the same is therefore true for $\pi_{1}(O(K, n))$.

Such a homomorphism $\phi$ as in Theorem 3.2 very often exists. For example, we shall show in Lemma 3.4 that this is always the case if $O$ is a complete, finite volume hyperbolic 3-orbifold. A variant of Theorem 3.2 is as follows.

Theorem 3.3. Let $O$ be a compact orientable 3-orbifold, and let $K$ be a knot in $O$ disjoint from its singular locus. Let $m$ be a positive integer such that $\pi_{1}(O(K, m))$ admits a surjective homomorphism $\phi$ onto a finite group $H$, with the property that $\phi(\langle[K]\rangle)$ has index at least $3 m$ in $H$. Then, for all sufficiently large $n$, $\pi_{1}(O(K, m n))$ is large.

Proof. This is very similar to the proof of Theorem 3.2. The kernel of $\phi$ corresponds to a finite regular covering map $\tilde{O} \rightarrow O(K, m)$. The inverse image of $K$ is a link $L$ in $\tilde{O}$, with at least 3 components, by our hypothesis on the index of $\phi(\langle[K]\rangle)$. Let $q$ be the order of any singularity along $L$, which we set to 1 if $L$ is disjoint from the singular locus of $\tilde{O}$. Let $\tilde{O}_{n}$ be the orbifold with the same underlying manifold as $\tilde{O}$, with singular locus consisting of that of $\tilde{O}$, but with a singularity of order $q n$ along $L$ (rather than $q$ ). We then have an induced covering map $\tilde{O}_{n} \rightarrow O(K, m n)$. Since $\pi_{1}\left(\tilde{O}_{n}\right)$ is large for all sufficiently large $n$, by Theorem 3.1 , the same is true of $\pi_{1}(O(K, m n))$ for all sufficiently large $n$.

The next result allows us to find homomorphisms $\phi$ as in Theorems 3.2 and 3.3 in most cases of interest.

Lemma 3.4. Let $G$ be a finitely generated, residually finite group that is not virtually cyclic. Then, for all $g \in G$ and any integer $N$, there is a surjective homomorphism $\phi$ from $G$ onto a finite group $H$ such that $[H: \phi(\langle g\rangle)]$ is at least $N$.

We will need the following elementary lemma.

Lemma 3.5. Let $G$ be a finitely generated, residually finite group that is not cyclic. Then, $G$ has a finite index characteristic subgroup $K$ such that $G / K$ is not cyclic.

Proof. Suppose, on the contrary, that, for every finite index characteristic subgroup $K$ of $G, G / K$ is cyclic. Then $G$ must be abelian. Otherwise, there are elements $g_{1}$ and $g_{2}$ of $G$ such that $\left[g_{1}, g_{2}\right] \neq e$. By the assumption that $G$ is residually finite, there is a finite index normal subgroup of $K_{1}$ of $G$ not containing $\left[g_{1}, g_{2}\right]$. Let $K$ be the intersection of the images of $K_{1}$ under all automorphisms of $G$. Then, $K$ is a finite index characteristic subgroup of $G$ not containing $\left[g_{1}, g_{2}\right]$. But, we are assuming that $G / K$ must be cyclic, which implies that $g_{1} K$ and $g_{2} K$ commute. Hence, $\left[g_{1}, g_{2}\right] K=K$, and so $\left[g_{1}, g_{2}\right] \in K$, a contradiction. Therefore, $G$ is a finitely generated abelian group. We will suppose that it is not cyclic and reach a 
contradiction. If it is finite, then $\{e\}$ is a finite index characteristic subgroup such that $G /\{e\}$ is not cyclic, which is a contradiction. If $G$ is infinite, then it is either of the form $\mathbb{Z}^{n}$, for some $n \geq 2$, or $\mathbb{Z} \times \mathbb{Z} / m \mathbb{Z} \times H$, for some integer $m \geq 2$ and some abelian group $H$. In both cases, set $K$ to be the subgroup generated by the $m$ th powers of $G$ (where $m=2$, say, in the former case) to achieve a contradiction.

Proof of Lemma 3.4. We prove this by induction on $N$. It is trivially true for $N=1$. Suppose, therefore, that $N$ is at least 2 and that the inductive hypothesis holds true for $N-1$. This implies that there is a surjective homomorphism $\phi$ from $G$ onto a finite group $H$ such that $[H: \phi(\langle g\rangle)]$ is at least $N-1$. Then, $K_{1}$, the kernel of $\phi$, is finitely generated, residually finite, and not cyclic. Therefore, by Lemma 3.5, there is a finite index characteristic subgroup $K$ of $K_{1}$ such that $K_{1} / K$ is not cyclic. Now, $K$ is a finite index normal subgroup of $G$. Let $\psi: G \rightarrow G / K$ be the quotient homomorphism. We claim that the index of $\psi(\langle g\rangle)$ in $G / K$ is at least $N$. This index is $[G:\langle g\rangle K]$, which equals $\left[G:\langle g\rangle K_{1}\right]\left[\langle g\rangle K_{1}:\langle g\rangle K\right]$. The first of these terms is, by assumption, at least $N-1$. It therefore suffices to show that $\left[\langle g\rangle K_{1}:\langle g\rangle K\right]$ is at least two. If this is not the case, then $\langle g\rangle K_{1}=\langle g\rangle K$. Taking intersections with $K_{1}$, we then deduce that $K_{1}=\langle g\rangle K \cap K_{1}$. This implies that $K_{1} / K$ is cyclic, generated by $g^{n} K$, where $g^{n}$ is a generator for $\langle g\rangle \cap K_{1}$. This is a contradiction.

Note that when $G$ is the fundamental group of a complete, finite volume hyperbolic 3-orbifold, then it satisfies the hypotheses of Lemma 3.4: it is finitely generated, residually finite and not virtually cyclic. Hence, Theorem 3.3 and Lemma 3.4 have the following corollary.

Theorem 3.6. Let $O$ be a compact orientable 3-orbifold (with possibly empty singular locus), and let $K$ be a knot in $O$, disjoint from its singular locus, such that $O-K$ has a complete, finite volume hyperbolic structure. Then, for infinitely many values of $n, \pi_{1}(O(K, n))$ is large.

Proof. It is a well known consequence of the proof of Thurston's hyperbolic Dehn surgery theorem [15] that for all sufficiently large $m, O(K, m)$ is hyperbolic. Hence, by Lemma 3.4, $\pi_{1}(O(K, m))$ admits a surjective homomorphism $\phi$ onto a finite group $H$, such that $\phi(\langle[K]\rangle)$ has index at least $3 m$ in $H$. Now apply Theorem 3.3 to deduce that $\pi_{1}(O(K, m n))$ is large for all sufficiently large $n$.

From the proof of the theorem, we obtain information about the set $(\Lambda$, say) of values of $n$ for which $\pi_{1}(O(K, n))$ is large. Specifically, there is an integer $A$ and, for each integer $m \geq A$, an integer $B(m)$ such that $\Lambda$ contains

$$
\{m n: m \geq A, n \geq B(m)\} .
$$

We now focus on a classical case: cyclic branched covers over a knot in the 3 -sphere.

Theorem 3.7. Let $K$ be a non-trivial knot in the 3-sphere, and let $m$ be any integer more than two. Then, for all sufficiently large $n$, the $m n$-fold cyclic cover of $S^{3}$ branched over $K$ has large fundamental group.

Proof. For any positive integer $n$, let $S^{3}(K, n)$ denote the orbifold with underlying manifold $S^{3}$ and with a singularity of order $n$ along $K$. Then the $n$-fold cyclic cover of $S^{3}$ branched over $K$ is a finite-sheeted covering space of $S^{3}(K, n)$. Our aim is therefore to show that $\pi_{1}\left(S^{3}(K, n)\right)$ is large for suitable values of $n$. 
Suppose first that $K$ is a connected sum of two non-trivial knots $K_{1}$ and $K_{2}$. Then $S^{3}(K, n)$ is an orbifold connected sum of $S^{3}\left(K_{1}, n\right)$ and $S^{3}\left(K_{2}, n\right)$. Now, $\pi_{1}\left(S^{3}\left(K_{1}, n\right)\right)$ and $\pi_{1}\left(S^{3}\left(K_{2}, n\right)\right)$ are quotients of $\pi_{1}\left(S^{3}(K, n)\right)$. Hence if one of these has large fundamental group, then so does $\pi_{1}\left(S^{3}(K, n)\right)$. So, it suffices to consider the case where $K$ is prime. This implies that $S^{3}(K, n)$ is (orbifold)-irreducible.

If $K$ is a satellite knot, then there is an essential torus in its complement. Since $K$ is prime, this remains (orbifold)-incompressible in $S^{3}(K, n)$, provided $n>1$. So, its inverse image $T$ in $M$, the $n$-fold cyclic branched cover of $S^{3}$ over $K$, is incompressible, provided $n>1$. Now, it is a theorem of Kojima 8 and Luecke [12] that if a compact orientable irreducible 3 -manifold $M$ contains essential embedded tori $T$, then either $\pi_{1}(M)$ is large or $M$ is finitely covered by a torus bundle over the circle, with $T$ lifting to fibres. We claim that the latter possibility cannot arise. This is because one component of the complement of $T$ covers a non-trivial knot exterior, and this component would be covered by $T^{2} \times I$. However, Theorem 10.5 of [6] implies that the only orientable irreducible 3 -manifolds that are finitely covered by $T^{2} \times I$ are $T^{2} \times I$ itself and the orientable twisted $I$-bundle over a Klein bottle. Therefore, $\pi_{1}\left(S^{3}(K, n)\right)$ is large when $n>1$.

If $K$ is a $(p, q)$-torus knot, then $S^{3}(K, n)$ is an orbifold Seifert fibre space with base orbifold that is topologically a sphere and has three singularities, with orders $p, q$ and $n$. When $n$ is more than $6,(1 / p)+(1 / q)+(1 / n)<1$, and therefore this base orbifold is hyperbolic. Its fundamental group is therefore large. But the Seifert fibration induces a surjective homomorphism from $\pi_{1}\left(S^{3}(K, n)\right)$ onto the fundamental group of this orbifold, and therefore $\pi_{1}\left(S^{3}(K, n)\right)$ is large, when $n>6$.

Thus, we may assume that $K$ is hyperbolic. Now, when $K$ is not the figureeight knot, it is a consequence of the Orbifold Theorem (2], Corollary 1.26 of 4]) that $S^{3}(K, m)$ is hyperbolic, when $m \geq 3$. So, by Lemma 3.4 and Theorem $3.3, \pi_{1}\left(S^{3}(K, m n)\right)$ is large, when $n$ is sufficiently large. When $K$ is the figure-eight knot, $S^{3}(K, m)$ is hyperbolic whenever $m \geq 4$, and so the theorem also holds in this case. However, $S^{3}(K, 3)$ is Euclidean. Its fundamental group is therefore residually finite and not virtually cyclic, and therefore Lemma 3.4 and Theorem 3.3 combine to prove the theorem here also.

It is natural to speculate whether Theorems 3.6 and 3.7 can be strengthened. Is it the case that when $K$ is a non-trivial knot in the 3 -sphere, $\pi_{1}\left(S^{3}(K, n)\right)$ is large for all but finitely many values of $n$ ? This remains an interesting unsolved problem. It suffices to consider the case where $n$ is prime, but this is the main situation where the arguments in this paper do not apply.

\section{REFERENCES}

[1] B. Baumslag and S. Pride, Groups with two more generators than relators, J. London Math. Soc. (2) 17 (1978), 425-426. MR0491967 (58:11137)

[2] M. Boileau and J. Porti, Geometrization of 3-orbifolds of cyclic type, Astérisque, vol. 272, 2001. MR1844891 (2002f:57034)

[3] J. Button, Strong Tits alternatives for compact 3-manifolds with boundary, J. Pure Appl. Algebra 191 (2004), 89-98. MR2048308 (2005f:57002)

[4] D. Cooper, C. Hodgson, and S. Kerckhoff, Three-dimensional orbifolds and cone-manifolds, MSJ Memoirs, vol. 5, Mathematical Society of Japan, 2000. MR:1778789 (2002c:57027)

[5] D. Cooper, D. Long, and A. Reid, Essential closed surfaces in bounded 3-manifolds, J. Amer. Math. Soc. 10 (1997), 553-563. MR1431827 (97m:57021) 
[6] J. Hempel, 3-Manifolds, Ann. of Math. Studies, vol. 86, Princeton University Press, 1976. MR0415619 (54:3702)

[7] J. Howie, Free subgroups in groups of small deficiency, J. Group Theory 1 (1998), 95-112. MR.1490160 (99e:20041)

[8] S. Kojima, Finite covers of 3-manifolds containing essential surface of Euler characteristic = 0, Proc. Amer. Math. Soc. 101 (1987), 743-747. MR911044 (89b:57010)

[9] M. Lackenby, A characterisation of large finitely presented groups, J. Algebra 287 (2005), 458-473. MR2134155 (2006a:20048)

[10] D. Long, Immersions and embeddings of totally geodesic surfaces, Bull. London Math. Soc. 19 (1987), 481-484. MR898729 (89g:57014)

[11] D. Long and G. Niblo, Subgroup separability and 3-manifold groups, Math. Z. 207 (1991), 209-215. MR:1109662 (92g:20047)

[12] J. Luecke, Finite covers of 3-manifolds containing essential tori, Trans. Amer. Math. Soc. 310 (1988), 381-391. MR965759 (90c:57011)

[13] J. Morgan, On Thurston's uniformization theorem for three-dimensional manifolds, The Smith conjecture, Pure Appl. Math., vol. 112, Academic Press, 1979, pp. 37-125. MR758464

[14] J. Ratcliffe, Euler characteristics of 3-manifold groups and discrete subgroups of $\mathrm{SL}(2, C)$, J. Pure Appl. Algebra 44 (1987), 303-314. MR885114 (88f:22028)

[15] W. Thurston, The geometry and topology of 3-manifolds (lecture notes), Princeton, 1978.

Mathematical Institute, University of Oxford, 24-29 St Giles, Oxford OX1 3LB, United Kingdom

E-mail address: lackenby@maths.ox.ac.uk 